\title{
Extracapsular Hip Fracture Management of a Patient Infected with COVID-19: A Novel Case Report
}

\author{
D Mustafa Yerli, (D) Serhat Gürbüz, (D) Ahmet Keskin, (D) Hakan Gürbüz
}

University of Health Sciences Turkey, Prof. Dr. Cemil Taşçıŏlu City Hospital, Clinic of Orthopedics and Trauma, Istanbul, Turkey

\section{Abstract}

The timing surgical treatment of a hip fracture on a patient with Coronavirus Disease-2019 (COVID-19) related pneumonia is determined based on the general condition of the patient and the circumstance of active disease in the lung. The common rule of earlier surgery is better motto, which is valid under normal conditions, can be stretched during the pandemic period, especially if the patient is infected with COVID-19. However, about the timing of surgery, attention should be paid to the patient's active lung infection condition in order to prevent facilitating the spread of the virus in the lung during intubation.

Keywords: COVID-19, SARS-CoV-2, intubation, intertrochanteric femur fracture, hip fracture, timing of surgery

\section{INTRODUCTION}

Coronaviridae virus family member Severe Acute Respiratory syndrome (SARS), also known as Coronavirus Disease-2019 (COVID-19), is a pandemic outbreak. Viruses of the family have a single-strand, positive-sense RNA genome, ranging from 26 to 32 kilobases in length (1). Among several coronaviruses pathogenic to humans, most are associated with mild clinical symptoms, with two notable exceptions: (a) SARScoronavirus-2 (SARS-CoV-2), which is a novel beta coronavirus that emerged in Guangdong, southern China, in November 2002, and resulted in more than 8,000 human infections and 774 deaths in 37 countries from 2002 to 2003, and (b) the Middle East Respiratory Syndrome-CoV-2, which was first detected in Saudi Arabia in 2012 and was responsible for 2,494 laboratory-confirmed infection cases and 858 fatalities since September 2012 (2-4). In late December 2019, several patients with viral pneumonia were epidemiologically associated with the Huanan seafood market in Wuhan, in China's Hubei province, where several nonaquatic animals such as birds and rabbits were for sale before the outbreak. A novel, human-infecting coronavirus, provisionally named 2019 novel coronavirus, was identified using next-generation sequencing (4-7). On March 11, 2020, the first case of the coronavirus pandemic was seen in Turkey according to the Turkish Ministry of Health. As time passed, the number of cases has dramatically increased in Turkey, which eventually declared an epidemic, and in many other countries. On the other hand, hip fractures are common among elderly adults, and these are generally related to osteoporosis. The two main types of hip fractures are femoral neck and intertrochanteric region proximal femoral fractures. Transtrochanteric femur fractures are usually common in older patients and are related to femoral neck fractures. However, patients infected with SARS-CoV-2 can experience concomitant health problems. In this case, a patient was infected with coronavirus, which was incidentally found in the hospital while being referred to the orthopedic clinic by the emergency department because of intertrochanteric region proximal femur fracture.

Phone: +90 5056073804 E-mail: mustafayerli199@gmail.com ORCID ID: orcid.org/0000-0002-2708-5812

Cite this article as: Yerli M, Gürbüz S, Keskin A, Gürbüz H. Extracapsular Hip Fracture Management of a Patient Infected with COVID-19: A Novel Case Report. Eur Arch Med Res 2020;36(4):289-93

๑Copyright 2020 by the University of Health Sciences Turkey, Prof. Dr. Cemil Taşçığlu City Hospital

European Archives of Medical Research published by Galenos Publishing House. 


\section{CASE PRESENTATION}

A 70-year-old male patient was admitted to the emergency department with a complaint of left hip pain after a fall. His wife was also admitted with symptoms of dyspnea and high fever. The coronavirus outbreak affects large areas, and every patient in the risk group who is admitted to our emergency room is assumed to be infected with SARS-CoV-2. Therefore, emergency service doctors have examined the patient and requested a thorax computed tomography (CT) scan for both our patient and his wife. The husband was referred to the orthopedic outpatient clinic from our emergency service with suspected left hip fracture.

Results from the CT scan revealed left extracapsular proximal femoral fracture and a chest finding that was suggestive of SARS-CoV-2 infection (Figure 1). An urinary catheter was inserted, and above-the-knee surgical stockings were applied on both legs to prevent embolization and urinary retention. He had no diagnosed morbidity but had a history of plastic surgery for a basal cell carcinoma in the facial region 6 years ago. We placed the patient in a temporary trauma observation room in our emergency department. Treatment was started immediately with subcutaneous enoxaparin sodium $60 \mathrm{mg}$ twice a day (equivalent to 6000 anti-Xa molecules each), $0.9 \%$ sodium chloride infusion at $100 \mathrm{~mL} / \mathrm{h}$ daily, intravenous omeprazole 40 mg once a day, and intravenous paracetamol $1000 \mathrm{mg}$ four times a day (8). Moreover, intravenous tramadol $100 \mathrm{mg}$ was added to his daily treatment chart and can be given as needed up to three times a day. The treatment regimen continued until discharge. Furthermore, the patient received respiratory physiotherapy three times a day for at least $10 \mathrm{~min}$ in the clinic.

Immobilization in elderly can cause severe problems such as delirium, venous embolization, electrolyte imbalances, urinary tract infection, depression, and muscle atrophy. Hence, urgent fixation surgery and rapid mobilization after surgery are required for elderly with hip fractures. During regular schedules,

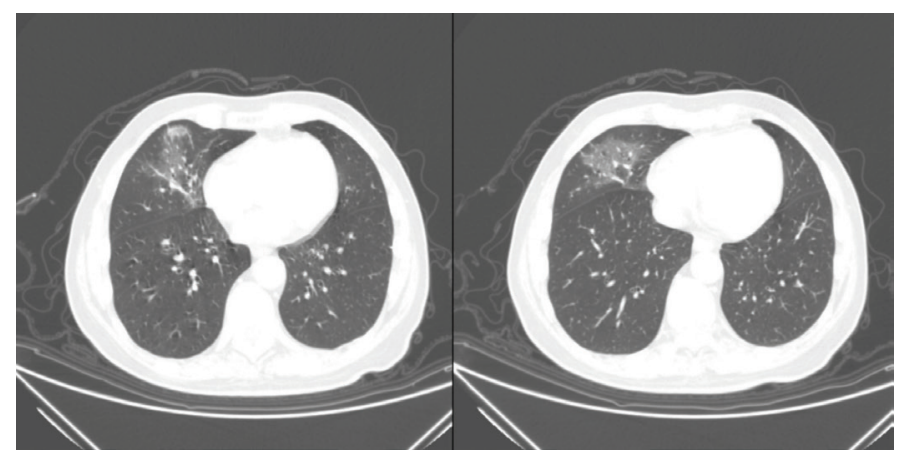

Figure 1. Thorax computed tomography of the patient on the day of admission our orthopedic and trauma clinic performs hip fracture surgery in elderly patients on the day after admission or $48 \mathrm{~h}$ subsequent to admission at maximum.

Generally, we admit patients in the hospital after checking their full blood count and giving their first medical treatment doses. However, in this case, the patient was first referred to the infectious diseases clinic because of suspicious thorax CT scan signs and pulmonary examination. In addition, the patient also had an uncertain contact history with SARS-CoV-2 infected people.

An infectious diseases and clinical microbiology consultant evaluated the patient, and a suspected SARS-CoV-2 related pneumonia was unveiled. After hospital admission, the infectious diseases and clinical microbiology requested again for surgical procedure availability and further suggestions. Consulting specialists recommended a few days' rest before surgery for the lungs to stabilize and infection to recede because of the high risk of intubation and mechanical ventilation, which will worsen the pneumonia. In our hospital, spinal anesthesia is used for hip fractures. However, the anesthesiology department did not want to risk as they did not have the option to shift to general anesthesia as consultations from the infectious diseases and clinical microbiology and pulmonology revealed that his lung condition can worsen after intubation and mechanical ventilation. Thus, the surgery was postponed.

In our hospital, patients infected with SARS-CoV-2 are placed in special isolation rooms. The patient and his wife were placed in the same isolation room; both were assumed to be SARS-CoV-2 positive. Both patients had thorax CT scans highly compatible with SARS-CoV-2 related pneumonia. Nasopharyngeal swab samples were instantly collected for real-time polymerase chain reaction $(\mathrm{PCR})$ tests at hospitalization. On admission, the patient had a $37.0{ }^{\circ} \mathrm{C}$ temperature, 84 beats per minute heart rate, 166/100 blood pressure, and 97\% oxygen saturation on room air. Oral hydroxychloroquine sulfate for 5 days (initial dose of oral $400 \mathrm{mg}$ twice a day peroral on the first day, then maintenance dose $200 \mathrm{mg}$ twice a day peroral 4 days) and intravenous ceftriaxone $1 \mathrm{~g}$ three times a day for 7 days for pneumonia and oral clarithromycin $500 \mathrm{mg}$ twice a day for 7 days for infection were added in the treatment chart. All doses of oral hydroxychloroquine were taken with a glass of milk. Therapy for SARS-CoV-2 pneumonia proceeded relentlessly, and anticoagulants, pain killers, proton pump inhibitors, and physiotherapy were continued. On the third day, the patient's PCR test came out negative, but his wife had a positive PCR test result. So, the treatment remained unaltered (9). 
During the entire hospitalization period, temperature, heart rate, respiration rate, and peripheral blood oxygen saturation were checked every hour. Daily electrocardiogram was done and QT interval calculated. Cognitive status was checked every day using the Memorial Delirium Assessment scale: no dysdiadochokinesia, no dyskinesia, no delirium, and no agitation were seen (10). The patient had a perpetual cough on the first two days and high fever $\left(38.6{ }^{\circ} \mathrm{C}\right)$ on the first day. Daily urinary output and liquid intake, including $2,400 \mathrm{~mL}$ intravenous solution of $0.9 \%$ sodium chloride, were recorded. Balanced total liquid intake and output were targeted. Besides, full blood count and blood biochemistry were checked daily to prevent hyperchloremic metabolic acidosis.

Consequently, minimal side effects were seen. Headaches were present on the first two days, but he had no diarrhea or rigid constipation. Even so, we advised a fibrous-weighted diet.

After the treatment was completed, approval for surgery was granted by both clinical microbiology and infectious diseases and pulmonology specialists. Thus, the patient went for surgery the day after. Informed consent was taken from the patient the day before operation; both the patient and his family were given detailed information about the procedure and possible complications.

There is a controversial debate on the gold standard treatment of extracapsular proximal femoral fractures. In our clinic, we prefer to perform intramedullary hip screw fixation for all A0 31-A type fractures (11), and this was done in this patient (Figures 2 and 3).

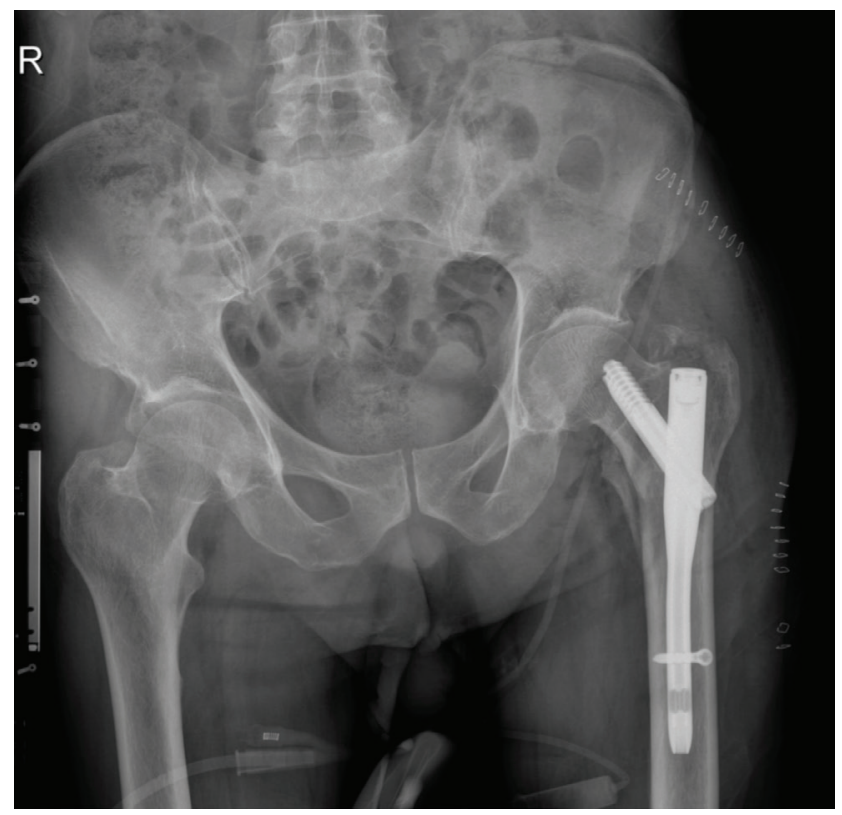

Figure 2. Anteroposterior radiograph of the patient's pelvis at first day postoperation
During surgery, each surgical staff wore a surgical mask as a cover of filtering facepiece class 3 mask and donned a transparent face shield on top. Moreover, they wore two pairs of sterile surgical apron and a lead apron on the innermost to shield them from the C-arm image intensifier radiation. At the bottom, all staff members wore rain boots, which are used only in surgery. Following the advice of the Turkish Ministry of Health, the A-M-G-H abbreviation was followed as a sequence for donning personal protective equipment: aprons, masks, glasses or face shields, and gloves.

The surgery lasted for $1 \mathrm{~h}$ and $15 \mathrm{~min}$, from skin incision to bandage. The image intensifier was used during the surgery. Although the surgical team was prompt, it took them longer than usual to take perioperative image intensifier shots. The operating team was composed of two surgeons (one orthopedic consultant and one fourth-year orthopedic resident), one surgical nurse, one anesthesiology technician, one anesthesiology consultant, and one operation room janitor. The aim was to perform the surgical procedure with maximal productivity and minimal complication.

A day after surgery, the patient started to mobilize with supervision from the orthopedic physiotherapist. Whole weightbearing mobilization was performed. Vitamin D, calcium, and zinc supplements were started for support. Discharge planning was difficult because of the recent surgery, but the patient's C-reactive protein and leukocyte count were controlled, and his subsequent thorax CT showed relevant healing results (Figure 4).

Consequently, the patient was informed about the rehabilitation process and given detailed instructions about beneficial actions for recovery. The patient and his family were informed about this study before discharge, and informed consent was obtained. Eventually, our patient was discharged from the hospital on the 15th day after his isolation period. Unfortunately, the patient's

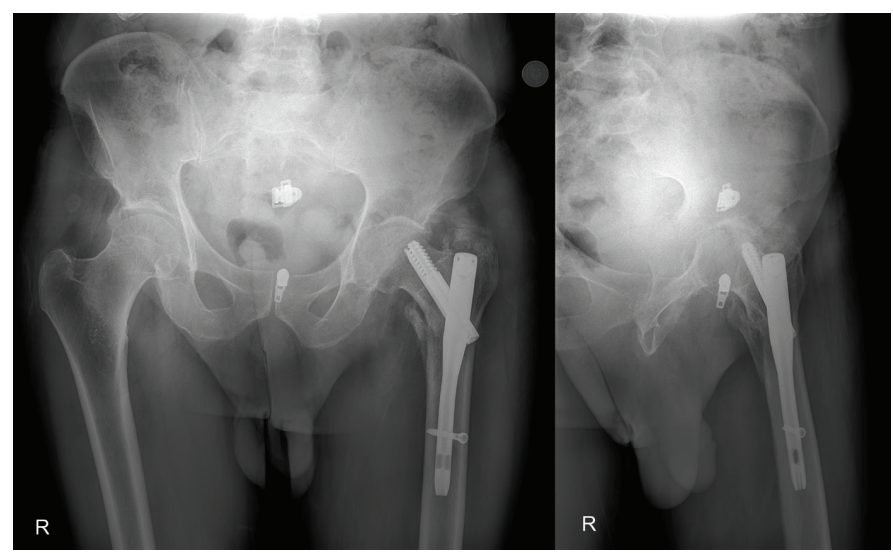

Figure 3. Pelvis anteroposterior and L-femur lateral radiographs of the patient at postoperative 6 weeks 


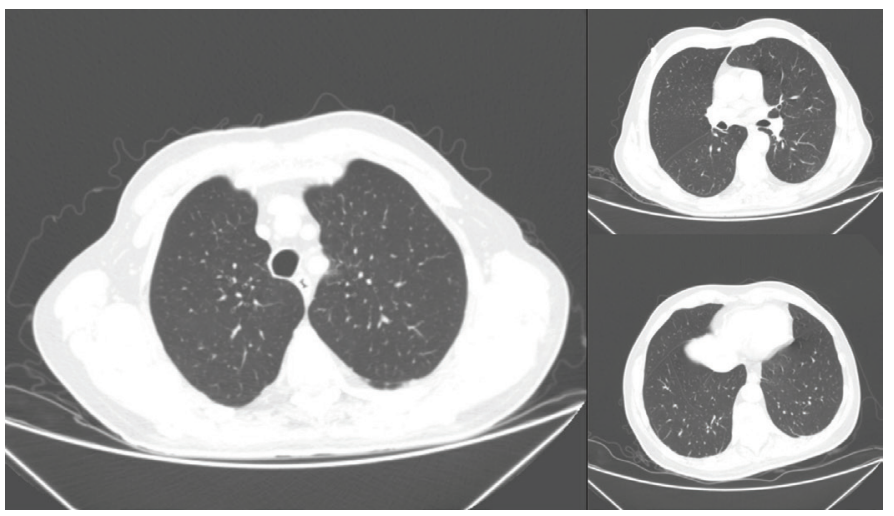

Figure 4. Patient's thorax computed tomography scans on the $14^{\text {th }}$ day of hospitalization

wife died in the intensive care unit because of acute respiratory distress syndrome due to SARS-CoV-2.

\section{DISCUSSION}

Many published articles on hip fractures in elderly people stated that early surgical treatment in the first $24 \mathrm{~h}$ is beneficial to reduce the rate of perioperative complications and mortality (12).

However, circumstances are not always appropriate for early surgery (13). These inconvenient conditions are sometimes due to the patient's general health or, in the current times, to the hospital's conditions. In this case study, the patient initially presented with a highly contagious pandemic viral infection SARS-CoV-2. The patient had viral pneumonia, and his respiratory system and vitals were strictly observed. After treating pneumonia, the patient was operated for hip fracture under firm precautions for healthcare workers. Although our patient was admitted together with his wife, he seemed to be luckier than her as he was discharged from the hospital after 15 days. The Turkey Ministry of Health demands an isolation period of 14 days for individuals infected or suspected of SARS-CoV-2. Thus, the patient has completed the isolation period.

To conclude, an outweighed conservative orthopedic treatment is preferred in these pandemic days (14). A surgical procedure performed as soon as possible and short hospitalization period are preferred to minimize contagious risks (12).

In managing trauma patients infected with SARS-CoV2, precautious treatment protocols are in place to preserve the well-being of both patients and healthcare workers. For us, the aim is to rapidly operate elderly with hip fractures; however, in our opinion, delaying surgery does not affect mortality if the patient has a condition that needs to be resolved preoperatively (15).

\section{CONCLUSION}

Finally, as we reported in this case, we consider that delaying surgery for selected trauma patients infected with SARS-CoV2 is the best option.

\section{Ethics}

Informed Consent: Informed consent was taken from the patient the day before operation.

Peer-review: Externally and internally peer-reviewed.

\section{Authorship Contributions}

Surgical and Medical Practices: M.Y., S.G., A.K., H.G., Concept: M.Y., S.G., A.K., H.G., Design: M.Y., S.G., A.K., H.G., Data Collection or Processing: M.Y., S.G., A.K., Analysis or Interpretation: M.Y., S.G., A.K., H.G., Literature Search: M.Y., S.G., A.K., Writing: M.Y., S.G., A.K.

Conflict of Interest: No conflict of interest was declared by the authors.

Financial Disclosure: The authors declared that this study received no financial support.

\section{REFERENCES}

1. Su S, Wong G, Shi W, Liu J, Lai ACK, Zhou J, et al. Epidemiology, Genetic Recombination, and Pathogenesis of Coronaviruses. Trends Microbiol 2016;24:490-502.

2. Peiris JSM, Guan Y, Yuen KY. Severe acute respiratory syndrome. Nat Med 2004;10:88-97.

3. Chan-Yeung M, Xu RH. SARS: epidemiology. Respirology 2003;8(Suppl 1):9-14.

4. Zaki AM, Boheemen S, Bestebroer TM, Osterhaus AD, Fouchier RA Isolation of a novel coronavirus from a man with pneumonia in Saudi Arabia. N Engl J Med 2012;367:1814-20.

5. Tan W, Zhao X, Ma X, Wang W, Niu P, Xu W, et al. A novel coronavirus genome identified in a cluster of pneumonia cases-Wuhan, China 20192020. China CDC Weekly 2020;2:61-2.

6. Zhu N, Zhang D, Wang W, Li X, Yang B, Song J, et al. A novel coronavirus from patients with pneumonia in China, 2019. N Engl J Med 2020;382:727-33.

7. Lu R, Zhao X, Li J, Niu P, Yang B, Wu H, et al. Genomic characterisation and epidemiology of 2019 novel coronavirus: implications for virus origins and receptor binding. Lancet 2020;395:565-74.

8. Falck-Ytter Y, Francis CW, Johanson NA, Curley C, Dahl OE, Schulman S, et al. Prevention of VTE in Orthopedic Surgery Patients: Antithrombotic Therapy and Prevention of thrombosis, 9th Ed: American Collage of Chest Physicians Evidence-Based Clinical Practice Guidelines. Chest 2012;141:e278-325. 
9. Long $\mathrm{C}, \mathrm{Xu} \mathrm{H}$, Shen Q, Zhang X, Fan B, Wang C, et al. Diagnosis of the coronavirus disease (COVID-19): rRT-PCR or CT? Eur J Radiol 2020;126:108961.

10. Breitbart W, Rosenfeld B, Roth A, Smith MJ, Cohen K, Passik S. The Memorial Delirium Assessment Scale. J Pain Symptom Manage 1997;13:128-37.

11. Crijns TJ, Janssen SJ, Davis JT, Ring D, Sanchez HB; Science of Variation Group. Reliability of the classification of proximal femur fractures: Does clinical experience matter? Injury 2018;49:819-23. doi: 10.1016/j. injury.2018.02.023. Epub 2018 Mar 15. Erratum in: Injury 2019;50:1266.

12. Pincus D, Ravi B, Wasserstein D, Huang A, Paterson JM, Nathens AB, et al. Association between wait time and 30-day mortality in adults undergoing hip fracture surgery. JAMA 2017;318:1994-2003.
13. Catellani F, Coscione A, D’Ambrosi R, Usai L, Roscitano C, Fiorentino G. Treatment of Proximal Femoral Fragility Fractures in Patients with COVID-19 During the SARS-CoV-2 Outbreak in Northern Italy. J Bone Joint Surg Am 2020;102:e58.

14. Halim A, Grauer JN. Orthopedics in the Era of COVID-19. Orthopedics 2020;43:138-9.

15. Seong YJ, Shin WC, Moon NH, Suh KT. Timing of hip fracture surgery in elderly patients: literature review and recommendations. Hip Pelvis 2020;32:11-6. 